\title{
ORDER AND COMMUTATIVITY IN BANACH ALGEBRAS
}

\author{
PHILIP C. CURTIS, JR.
}

S. Sherman has shown [4] that if the self adjoint elements of a $C^{*}$ algebra form a lattice under their natural ordering the algebra is necessarily commutative. In this note we extend this result to real Banach algebras with an identity and arbitrary Banach * algebras with an identity. The central fact for a real Banach algebra $A$ is that if the positive cone is defined to be the uniform closure of the set of finite sums of squares of elements of $A$, and if $A$ is a lattice under the ordering induced by this cone, then extreme points of the unit sphere of the dual cone are multiplicative linear functionals. A similar situation holds for ${ }^{*}$ algebras.

1. Real Banach algebras. Let $X$ be a real Banach space, and let $C$ be a closed cone in $X .{ }^{1}$ For $x, y \in X$ we define $x \geqq y$ if $x-y \in C$. If in addition $X$ is a lattice under the ordering $\geqq$, we say $C$ latticeorders $X$. Let $C^{\prime}$ be the dual cone and let $\sum=\left\{f \in C^{\prime}:\|f\| \leqq 1\right\}$. The set of extreme points of $\sum$ will be denoted by $S$. For a real linear functional $f$ let $I_{f}=\{x \in X: f(x)=0\}$ and let $R=\bigcap_{f \in C^{\prime}} I_{f}$. Lastly if $X$ is a lattice we define $x_{+}=x \vee 0, x_{-}=x \wedge 0$, and $|x|=x_{+}-x_{-}$. We note $|x| \geqq 0$.

Lemma 1. If $C$ is a closed cone in a real Banach space $X$, then

(i) $\mathrm{R}=\mathrm{C} \cap-\mathrm{C}$,

(ii) $R=\bigcap_{f \in S} I_{f}$,

(iii) If C lattice-orders $X, R=\{0\}$.

Proof. Obviously $C \cap-C \subset R$. For the converse, by the HahnBanach theorem $x \in C$ iff $f(x) \geqq 0$ for each $f \in C^{\prime}$. Therefore $R \subset C \cap$ $-C$. For (ii) suppose $x \in \bigcap_{f \in S} I_{f}, x \in R$, then there exists an $f \in C^{\prime}$, $\|f\|=1$, such that $|f(x)|=2 \epsilon \neq 0$. But by the Krein-Milman theorem there exist finitely many $f_{i} \in S$ and real numbers $\alpha_{i}$ such that $\left|f(x)-\sum \alpha_{i} f_{i}(x)\right|<\epsilon$. Hence for some $i, f_{i}(x) \neq 0$, which is a contradiction. Lastly let $C$ lattice order $X$. Then $x \in C$ implies $x \geqq 0$ or $x_{-}=0$, and $x \in-C$ implies $-x \geqq 0$ or $x_{+}=0$. Since $x=x_{+}+x_{-}$, $x \in C \cap-C$ implies $x=0$.

The central tool in both this investigation and that of Sherman is the following result of Krein and Krein [3]. It can be stated in a

Received by the editors August 5, 1957.

1 We refer the reader to [2] for the appropriate definitions of cone, dual cone, etc. 
slightly more general fashion, but the following is sufficient for our purposes.

THEOREM 1. Let $X$ be a real Banach space which is lattice ordered by a cone $C$. Suppose in addition that $C$ contains an element $e,\|e\|=1$, such that $\{y:\|e-y\| \leqq 1\} \subset C$. Then $f \in S$ iff $|f(x)|=f(|x|)$ for each $x \in X$.

Let us specialize to a real Banach algebra $A$ with identity such that $\|1\|=1$, and let $C$ be the closure of the set of finite sums of squares of elements of $A$. By the familiar binomial series argument (c.f. [2]), $\{y:\|1-y\| \leqq 1\} \subset C$. Also, for $f \in C^{\prime}$ and $x, y \in A$ we have the Schwartz inequality, $[f(x y+y x)]^{2} \leqq 4 f\left(x^{2}\right) f\left(y^{2}\right)$, which may be verified by the classical argument. Also useful is the following property of functionals in $C^{\prime}$.

Lemma 2. Let $A$ be a real Banach algebra, and let $x \in C, f \in C^{\prime}$. Then $f(x)=0$ implies $f\left(x^{2}\right)=0$.

Proof. First assume $x=y^{2}$, and let $f \in C^{\prime}$. If $\|x\| \leqq 1$, the binomial series for $(1-x)^{1 / 2}$ converges absolutely. Therefore $1-x \in C$. Moreover since $x=y^{2}, y(1-x)^{1 / 2}=(1-x)^{1 / 2} y$ and $x(1-x)=\left[y(1-x)^{1 / 2}\right]^{2}$ $\in C$. Therefore $x-x^{2} \geqq 0$. Hence $f(x)=0$ implies $f\left(x^{2}\right)=0$. We proceed now by induction. Let $x=\sum_{i=1}^{n+1} y_{i}^{2}$ and let $f(x)=0$. If $z=\sum_{i=1}^{n} y_{i}^{2}$, then $f\left(y_{n+1}^{2}\right)=f(z)=0$. We assume $f\left(z^{2}\right)=0$, and by the above argument $f\left(y_{n+1}^{4}\right)=0$. An application of the Schwartz inequality gives us

$$
0 \leqq f\left(x^{2}\right)=f\left(\left(z+y_{n+1}^{2}\right)^{2}\right)=f\left(z y_{n+1}^{2}+y_{n+1}^{2} z\right) \leqq 2\left[f\left(z^{2}\right) f\left(y_{n+1}^{4}\right)\right]^{1 / 2}=0 .
$$

Therefore the result holds for all finite sums of squares, and by continuity it holds for all $x$ in $C$.

Theorem 2. If $C$ lattice-order $A$ then each $f \in S$ is a homomorphism of $A$ onto the real numbers, and $A$ is commutative.

Proof. For $x, y \in A$ define the Jordan multiplication $x \circ y$ $=(x y+y x) / 2$. Thus $A$ can be considered as a Jordan ring with an identity. We assert that for $f \in S, I_{f}$ is a Jordan ideal. By Theorem 1 $x \in I_{f}$ iff $x_{+}, x_{-} \in I_{f}$. Therefore let $x \geqq 0, x \in I_{f}$. By the Schwartz inequality and Lemma 2 , for any $y \in A,[f(x y+y x)]^{2} \leqq 4 f\left(x^{2}\right) f\left(y^{2}\right)=0$. Hence $x y+y x \in I_{f}$, and since $I_{f}$ is obviously closed under addition, $I_{f}$ is a Jordan ideal.

Now a linear functional of any algebra over a field which takes the identity of the algebra into the identity of the field is a homomorphism if its kernel is a two-sided ideal. Hence $f$ is a Jordan homo- 
morphism of $A$ onto the reals. On the other hand Jacobson and Rickart [1, Theorem 2] have proved that a Jordan homomorphism of a ring into an integral domain is either a homomorphism or an antihomomorphism. An application of this result proves that $f$ is a homomorphism.

Finally for each $f \in S$ and $x, y \in A, x y-y x \in I_{f}$. Since by Lemma 1 $\bigcap_{f \in S} I_{f}=\{0\}, A$ must be commutative.

2. Banach * algebras. Let $A$ be a Banach * algebra with a continuous involution and an identity. Let $C$ be the closure of the set of finite sums of elements $x x^{*}$. $C$ is a closed cone in the real linear space $H$ of self adjoint elements of $A$. The dual cone of $C$ (in the conjugate space of $H$ ) can be identified with the set of those functionals $f$ on $A$ for which $f\left(x x^{*}\right) \geqq 0$ for each $x \in A$ (c.f. [2] for details). Let $\sum, S$ be as before and for $f \in C^{\prime}$ let $I_{f}=\{x \in A: f(x)=0\}$ and $R=\bigcap_{f \in C^{\prime}} I_{f}$. We also note that $\{h \in H:\|1-h\| \leqq 1\} \subset C$ and for $f \in C^{\prime}$ the familiar Schwartz inequality holds, i.e. $\left|f\left(x y^{*}\right)\right|^{2} \leqq f\left(x x^{*}\right) f\left(y y^{*}\right), x, y \in A$.

LEMMA 3.

$$
\begin{aligned}
& R=C \cap-C+i(C \cap-C), \\
& R=\bigcap_{f \in S} I_{f} .
\end{aligned}
$$

Proof. Let $T=(C \cap-C)+i(C \cap-C)$. Obviously $T \subset R$. If $x \in R$, let $h=\left(x+x^{*}\right) / 2, k=\left(x-x^{*}\right) / 2 i$. Then $h, k$ are self adjoint, $h, k \in I_{f}$ and $x=h+i k$. But a self adjoint element $y \in C$ iff $f(y) \geqq 0$ for each $f \in C^{\prime}$. Therefore $h, k \in C \cap-C$ and $T=R$. For the second assertion if $x \in \bigcap_{f \in S} I_{f}$ and $x \in R$, we may assume $x$ is self adjoint and apply the argument of Lemma 1.

Lemma 4. Let $h \in C$ and $f \in C^{\prime}$. Then $f(h)=0$ implies $f\left(h^{2}\right)=0$.

Proof. If $h=h^{*}$, and $\|h\| \leqq 1$, then by the familiar series argument $1-h=k^{2}$, where $k=k^{*}$. Therefore $1-h \in C$. Since $k h=h k$ and $k h k \in C$, $k h k=h k^{2}=h-h^{2} \in C$. Therefore $f(h)=0$ implies $f\left(h^{2}\right)=0$.

Theorem 3. If $C$ lattice-orders $H$, then each $f \in S$ is a homomorphism of $A$ onto the complex numbers, and $A$ is commutative.

Proof. To prove that $f$ is a homomorphism it suffices to show that for $f \in S, I_{f}$ is a two-sided ideal. Let $x \in I_{f}, y \in A$. We assert $x y \in I_{f}$. First we may assume $x$ is self adjoint and by Theorem 1 we may assume $x \geqq 0$. But then applying the Schwartz inequality

$$
|f(x y)|^{2} \leqq f\left(x^{2}\right) f\left(y^{*} y\right)=0 .
$$


This proves $x y \in A$ and similarly $y x \in A$. Since $I_{f}$ is obviously closed under addition, it is a two sided ideal. An application of Lemma 3 proves that $A$ is commutative.

\section{BIBLIOGRAPHY}

1. N. Jacobson and C. E. Rickart, Jordan homomorphism of rings, Trans. Amer. Math. Soc. vol. 69 (1950) pp. 479-502.

2. J. L. Kelley and R. L. Vaught, The positive cone in Banach algebras, Trans. Amer. Math. Soc. vol. 74 (1953) pp. 44-55.

3. M. Krein and S. Krein, On an inner characteristic of the set of all continuous functions defined on a bicompact Hausdorff space, Comptes Rendus (Doklady) de l'Academie des Sciences de l'URSS. N. S. vol. 27 (1940) pp. 427-430.

4. S. Sherman, Order in operator algebras, Amer. J. Math. vol. 73 (1951) pp. 227232.

University of California at Los Angeles

\section{A NOTE ON VALUED LINEAR SPACES}

PAUL CONRAD

Banaschewski [1] has given a simple and elegant proof of Hahn's embedding theorem for ordered abelian groups. His method can be used to prove the author's generalization of Hahn's theorem [2, p. 11]. In this note we make use of Banaschewski's method to prove a special case of the author's theorem (which is also a generalization of Hahn's theorem) that has been proven by Gravett [3].

Let $(L, \Delta, d)$ be a valued linear space [3]. That is, $L$ is a vector space over a division ring $K, \Delta$ is a linearly ordered set with minimum element $\theta$, and $d$ is a mapping of $L$ onto $\Delta$ such that for all $x, y \in L$, $d(x)=\theta$ if and only if $x=0, d(x)=d(k x)$ for all $0 \neq k \in K$, and $d(x+y)$ $\leqq \operatorname{Max}[d(x), d(y)]$. For each $\delta \in \Delta$, let $C^{\delta}=\{x \in L: d(x) \leqq \delta\}$ and let $\bar{C}_{\delta}=\{x \in L: d(x)<\delta\}$. Let $W$ be the vector space of all mappings $f$ of $\Delta$ into the join of the spaces $C^{\delta} / C_{\delta}$ for which $f(\delta) \in C^{\delta} / C_{\delta}$ and $R_{f}=\left\{\delta \in \Delta: f(\delta) \neq C_{\delta}\right\}$ is an inversely well ordered set. $W$ is a subspace of the unrestricted direct sum $V$ of the $C^{\delta} / C_{\delta}$. W is also a valued linear space $\left(W, \Delta, d^{\prime}\right)$, with $d^{\prime}(f)$ the largest $\delta \in R(f)$.

Received by the editors February 1, 1958. 